\author{
Usa Thisyakorn* and Chule Thisyakorn \\ Department of Pediatrics, Faculty of Medicine, \\ Chulalongkorn University, Bangkok, Thailand \\ Dates: Received: 01 August, 2016; Accepted: 09 \\ August, 2016; Published: 10 August, 2016 \\ ${ }^{*}$ Corresponding author: Usa Thisyakorn, \\ Department of Pediatrics, Faculty of \\ Medicine, Chulalongkorn University, 1873 \\ Rama IV Road, Bangkok 10330, Thailand, \\ E-mail: uthisyakorn@gmail.com \\ www.peertechz.com \\ Keywords: Dengue; Vaccines; Development
}

\section{Research Article \\ Prospects for the Development of a Dengue Vaccine}

\section{Abbreviation}

DF: Dengue Fever; DHF: Dengue Hemorrhagic Fever; DSS: Dengue Shock Syndrome; Prm: Premembrane; M: Membrane; E: Envelope; C: Capsid; WHO: World Health Organization; CYD-TDV: Chimera Yellow Fever Dengue Tetravalent Dengue Vaccine; SAGE: Strategic Advisory Group of Experts; ADS: Asia Dengue Summit; ADVA: Asia Dengue Vaccination Advocacy; DVI: Dengue Vaccine Initiative; SEAMEO TROPMED: The Southeast Asian Ministers of Education Organization Tropical Medicine and Public Health; Fmx: Fondation Meriux; GDAC: Global Dengue and Aedestransmitted Diseases Consortium

\section{Introduction}

Dengue is a mosquito-borne viral disease which is currently an expanding global problem. Successful treatment, which is mainly supportive, depends on early recognition of the disease and careful monitoring for shock. A severity-based revised dengue classification for medical interventions has been developed by the World Health Organization and was adopted in most countries. Dengue is one disease entity with different clinical manifestations; often with unpredictable clinical evolutions and outcomes. Four closely related dengue serotypes cause the disease, which ranges from asymptomatic infection to undifferentiated fever, dengue fever (DF), and dengue hemorrhagic fever (DHF). The severity of DF manifestations increases with age. DF causes fever, rash, muscle or joint pain, headache, eye pain but is rarely fatal. DHF is considered a distinct disease characterized by fever, bleeding diathesis, and increased vascular permeability leading to leakage of plasma with a tendency to develop potentially fatal dengue shock syndrome (DSS). Although shock and plasma leakage seem to be more prevalent as age decreases, the frequency of internal hemorrhage rises as age increases. Increase in liver enzymes found in both children and adults indicates liver involvement during dengue infections. Pre-existing liver diseases which are more common in adults such as chronic hepatitis, alcoholic cirrhosis, and hemoglobinopathies can aggravate the liver impairment in dengue patients. Dengue with organ impairment mainly involves the liver and the central nervous system. Consistent hematological findings include vasculopathy, coagulopathy, and thrombocytopenia. Laboratory diagnosis includes virus isolation, serology, and detection of dengue ribonucleic acid. The age of dengue cases in several countries has increased from children to adolescents and adults. The pathogenesis of dengue infection is not well understood although antibody-dependent enhancement has been implicated in the pathogenesis [1].

Antiviral medications are not available for dengue and successful treatment, which is mainly supportive, depends on early recognition of the disease, bleeding tendency and careful monitoring for signs of circulatory failure. Adults have a higher prevalence of underlying diseases e.g. coronary artery disease, peptic ulcer, hypertension, diabetes mellitus, cirrhosis, or chronic kidney diseases, which should be considered in dengue management. A severity-based revised dengue classification for medical interventions has been developed and adopted in many countries [2]. Prevention using vector control has limited success, dengue vaccine is then seen as one of the major tools in effectively control dengue diseases [3,4].

\section{Dengue vaccine development}

Dengue virus is a positive-sense, single stranded, $11 \mathrm{~kb}$ RNA flavivirus consisting of three structural proteins (premembrane/ membrane (prM/M), envelope (E), and capsid (C) and seven nonstructural proteins. There are four antigenically distinct serotypes (DENV-1, 2, 3, and 4). The pathogenesis of DHF is not clearly 
understood, the uniqueness of the dengue viruses and the spectrum of diseases resulting from infection have made dengue vaccine development difficult. Several different approaches have been done to develop a dengue vaccine; since dengue is a unique and complex disease, developing a dengue vaccine has proven equally complex. However, there is a robust vaccine pipeline currently in clinical and preclinical studies including live attenuated vaccines, subunit, DNA purified inactivated vaccine candidates as well as virus-vectored and virus-like particle-based vaccines [5-7].

Inactivated vaccines have two advantages since they cannot revert to a more pathogenic phenotype and they are unlikely to interfere with each other in combination. Induction of cell-mediated and humoral immune responses has been demonstrated with inactivated vaccines. However, inactivated vaccines express only the part of the viral genome that encodes structural proteins. In the context of dengue immunity and immunopathogenesis, raising antibodies that may not be fully protective may lead to breakthrough infections or enhance infections with wild-type dengue viruses and a requirement of adjuvants for enhancing immunogenicity [5].

\section{Licensed dengue vaccine}

The first dengue vaccine, CYD-TDV (Dengvaxia, Sanofi Pasteur, Lyon, France), a liveattenuated tetravalent dengue vaccine with a yellow fever backbone and all four dengue serotype components are chimeric (prM and E proteins), has now been licensed by several dengue endemic countries in Asia and Latin America for using in persons aged 9-45 or 9-60 years, and is under regulatory review in several others [8].

The first Phase III efficacy trial for CYD-TDV in highly dengueendemic area of five Asian countries in 10,275 children demonstrated that this dengue vaccine is efficacious when given as a 0-6-12 month schedule to 2-14 year-old children. The vaccine showed a $56.5 \%$ (95\% CI: 43.8-66.4) overall efficacy with the contribution of each of the 4 serotypes, and more than $80 \%$ of severe dengue episodes were avoided with a two-third reduction in hospitalization. Higher efficacy was observed in the immunogenicity subset seropositive at baseline. Good safety profile was observed with an interesting finding that vaccine efficacy was higher for participants who were seropositive for dengue than for those who were seronegative. Furthermore, vaccine efficacy increased with age, which could be a marker of previous exposure to dengue [9].

A second Phase III clinical trial in Latin American countries in 20,875 children and adolescents aged 9-16 years demonstrated a $60.8 \%$ (95\% CI: 52.0-68.0) overall efficacy with the contribution of each of the 4 serotypes. Additional observation of the results showed a significant reduction of the risk of hospitalization by $80.3 \%$. Higher efficacy was observed in the immunogenicity subset seropositive at baseline with good safety profile [10]. The burdens of dengue were substantial in both regions and in all age groups. Burdens varied widely according to country, but the rates were generally higher and the disease more frequently severe in Asian countries than in Latin American countries [11].

Both trials have shown that the vaccine provided clinically important reductions in hospitalization and the comparison of the symptomatology of disease between vaccine and placebo recipients suggests that disease was milder after vaccination, without evidence of antibody dependent enhancement $[9,10]$.

The unexplained higher incidence of hospitalization for dengue in year 3 among those younger than 5 years children needs to be carefully monitored during long-term follow-up. Assessment of the incidence of hospitalization for virologically confirmed dengue as a surrogate safety end point during follow-up in years 3 to 6 of two phase III trials and a phase IIb trial showed the risk among children 2 to 16 years of age was lower in the vaccine group than in the control group [12].

The World Health Organization (WHO) Strategic Advisory Group of Experts (SAGE) on Immunization recommends countries consider introduction of CYD-TDV in geographic settings where dengue is highly prevalent. SAGE recommends that vaccination should be considered as an integrated strategy together with communication strategy, well-executed and sustained vector control, the best evidence-based clinical care for all patients with dengue, and robust dengue surveillance [8]. The observed vaccine efficacy against asymptomatic dengue infection is expected to translate into reduced dengue virus transmission, if sufficient individuals are vaccinated in endemic areas [13].

The World Health Organization (WHO) published in the Weekly Epidemiological Record its first position paper on a dengue vaccine, based on the available evidence of CYD-TDV or Dengvaxia, the only dengue vaccine to have received regulatory approval. Starting in December 2015, Dengvaxia has been approved by the regulatory authorities of Mexico, the Philippines, Brazil, El Salvador, Paraguay and Costa Rica. WHO recommends that countries should consider introduction of the dengue vaccine Dengvaxia only in geographic settings (national or subnational) where epidemiological data indicate a high burden of disease and dengue vaccine introduction be a part of a comprehensive dengue control strategy, including wellexecuted and sustained vector control, evidence-based best practices for clinical care for all patients with dengue illness and strong dengue surveillance [14].

\section{Dengue vaccine in the pipeline}

Two dengue vaccine candidates at advanced stages of clinical development are both live-attenuated vaccines and both have one or more chimeric serotype component. TDV (Takeda, Osaka, Japan) has one component that is attenuated but not chimeric (DEN-2) and three chimeric components (prM and E proteins); while TV003/ TV005 (National Institutes of Health, NIH, Bethesda, MD, USA) has three attenuated components and one chimeric component (DEN-4 backbone with DEN-2 prM and E proteins). Both are at Phase II and III efficacy trial stages [15].

The increasing knowledge of dengue vaccine development is providing more insights into improved vaccine design. Recent advances in vaccine science have greatly increased the technological options for dengue vaccine development as several promising dengue vaccine candidates are in preclinical and clinical development. In parallel, molecular biology and system biology permit more specific analysis of vaccine-induced immunogenicity and safety. However, 
possibly the most intriguing finding in relation to dengue vaccines over the past year comes from the first licensed CYD-TDV. There are also three inactivated whole virus dengue vaccines with incorporated adjuvants for enhancing immunogenicity at the Phase I trial stage. The preclinical dengue vaccine pipeline covers a broad range of approaches, both in relation to antigen as well as in relation to delivery and presentation. Second generation vaccines may improve upon first generation vaccines in relation to overall and strain-specific vaccine efficacy, in particular in immunologically naïve subjects, and more favorable immunization schedules. Carefully designed studies in nonhuman primates should allow prioritization of preclinical candidates for human subject trials $[4,5,7]$. Future clinical development of dengue vaccine candidates will need to consider the fact that CYD-TDV (Dengvaxia', Sanofi Pasteur, Lyon, France) is introduced into many endemic countries.

\section{Asia dengue summit}

During 13-14 January 2016, the Asia Dengue Summit (ADS) co-organized by the Asian Dengue Vaccination Advocacy (ADVA), the Dengue Vaccine Initiative (DVI), the Southeast Asian Ministers of Education Organization Tropical Medicine and Public Health Network (SEAMEO TROPMED) and the Fondation Mérieux (FMx) was held in Bangkok, Thailand and focused on improving strategies for dengue prevention and control. The ADS and the ADVA workshop presentations provided a foundation from which to form an outcome statement and call to action. Since dengue vaccine is a potentially game-changing tool and what happen in 2016 will impact the future for populations in dengue endemic areas around the world. The second part of the meeting comprised discussion of a set of statements drafted by ADVA based on the key messages from the $\mathrm{ADS}$ as the following:

\section{Call to Action}

\section{Within the broader context of the outcomes of the asia dengue summit}

We

- Recognize that dengue continues to be a major global public health threat and the problem is growing.

- Recognize that vaccines would be a useful addition to current prevention and control efforts which, in most cases, are inadequate for full impact.

- Are aware of the licensure of the first dengue vaccine and imminent availability of other promising candidates for preventive vaccination.

- Are cognizant of the strong leadership role provided by the WHO, guided by the Global Strategy for Dengue Prevention and Control, to reduce dengue mortality by $50 \%$ and morbidity by $25 \%$ by the year 2020 .

- Are informed by the urgent need for adequate resources, integrated surveillance, sustainable vector control methods, adequate preparedness for vaccination programmes, including school-based vaccination, appropriate evaluation and monitoring of interventions and need for new methodologies and high-quality point-of-care diagnostic tests.
- Recognize the central role of good science, good communications, the media, strong political leadership and public support.

- Are sensitive to the need to ensure equity, sustainability, ethics and social justice.

\section{The co-hosts of the asia dengue summit make the following call to action}

1. Based on close consideration of their own disease epidemiology, capacities, health infrastructure, financial resources and decision on vaccine registration, call on countries, where appropriate, to develop and implement a carefully controlled stepwise programmatic introduction of dengue vaccine(s), including school-based vaccination and catch-up campaigns, which are closely integrated with other control strategies and the needs and constraints of the health system.

2. Call on countries to ensure that vaccine implementation programmes are monitored and tracked, and evaluated for safety, effectiveness and acceptance through sound risk communication and management plans, with good communications, active surveillance, laboratory support and clinical management.

3. Guided by the need for better integration and synergy of strategies, and in order to avoid fragmentation and duplication, call on various related initiatives to work closely together through global efforts such as the Global Dengue and Aedestransmitted diseases Consortium (GDAC), including to monitor developments in vaccine and vector control implementation and to perform high-level advocacy with governments and international organizations for vaccine introduction in endemic countries.

4. Call for the political will and commitment to accelerate effective dengue prevention and control interventions, including strengthening health systems and ensuring sustainable financing.

5. Call on relevant organizations and institutions to continue performing and supporting research to further enhance the impact of vaccination, including biomedical and clinical research, mathematical modelling, implementation and operational research, and post-licensure studies.

6. Call on the WHO and other global and regional organizations and initiatives to give higher priority to dengue prevention and control, provide continued leadership, guidance and technical support to countries on the possible introduction of dengue vaccine, and assist with implementation strategies, as well as assisting countries in obtaining sustainable financing $[5,16]$.

\section{ASEAN dengue day}

ASEAN Dengue Day is an advocacy event held every 15 June to increase public awareness of dengue, to mobilize resources for its prevention and control, and to demonstrate the region's commitment 
to tackling the disease. The advocacy event was agreed upon during the 10th ASEAN Health Ministers Meeting in 2010. The first regional event was held in 2011 in Jakarta, Indonesia. During 13-15 June, 2016, the $6^{\text {th }}$ ASEAN Dengue Day was held in Bangkok, Thailand where health experts call for a collective regional action to fight the fastest-growing burden of dengue in ASEAN region [17].

\section{Conclusion}

The global burden of dengue is increasing rapidly, driven by population growth, urbanization, globalization, and ecological changes. A dengue vaccine is needed as part of an integrated approach to dengue prevention and control. One dengue vaccine, CYD-TDV has been licensed in several dengue endemic countries in Latin America and Asia since 2015 for use in 9-45 or 9-60year old individuals. Two other vaccines are at advanced stages of clinical development at Phase II and III. Several other vaccines are at varying stages of preclinical and clinical development.

\section{References}

1. Thisyakorn U, Thisyakorn C (2015) Dengue: Global Threat. Southeast Asian J Trop Med Public Health 46: 3-10.

2. Tantawichien $T$ (2015) Dengue fever and dengue haemorrhagic fever in adults. Southeast Asian J Trop Med Public Health 46: 79-98.

3. Horstick O, Ranzinger SR (2015) Interim analysis of the contribution of highlevel evidence for dengue vector control. Southeast Asian J Trop Med Public Health 46: 131-137.

4. Thisyakorn U, Thisyakorn C (2015) Dengue vaccines. Southeast Asian J Trop Med Public Health 46: 138-145.

5. Thisyakorn U. Thisyakorn C (2014) Latest developments and future directions in dengue vaccines. Ther Adv Vaccines 2: 3-9.
6. Prommalikit $\mathrm{O}$, Thisyakorn $\mathrm{U}(2015)$ Dengue virus virulence and diseases severity. Southeast Asian J Trop Med Public Health 46: 35- 42.

7. Vannice KS, Roehrig JT, Hombach J (2016) Next generation dengue vaccines: A review of the preclinical development pipeline. Vaccine 33: 7091 7099.

8. World Health Organization (2016) Dengue vaccine. Weekly epidemiological record 21: 282-284

9. Capeding MR, Tran NH, Hadinegoro SRS, Ismail HIM, Chotpitayasunondh $T$, et al. (2014) Clinical efficacy and safety of a novel tetravalent dengue vaccine in healthy children aged 2 to 14 years in Asia: a phase III randomized observer-masked, placebo-controlled trial. Lancet 384: 1358-1365.

10. Villar L, Dayan GH, Arredondo-Garcia JL, Rivera DM, Cunha R, et al. (2015) Efficacy of a tetravalent dengue vaccine in children in Latin America. $\mathrm{N}$ Engl J Med 372: 113-123.

11. L'Azou M, Moureax A, Sarti E, Nealon J, Zambrano B, et al. (2016) Symptomatic Dengue in Children in 10 Asian and Latin American Countries. N Engl J Med 374: 1155-1166.

12. Hadinegoro SR, Arredondo-Garcia JL, Capeding MR, Deseda C, Chotpitayasunondh T, et al. (2015) Efficacy and longterm safety of a dengue vaccine in region of endemic disease. $\mathrm{N}$ Engl J Med 373: 1195-1206.

13. Olivera-Botello G, Coudeville L, Fanouillere K, Guy B, Chambonneau L, et al. (2016) Tetravalent dengue vaccine reduces symptomatic and asymptomatic dengue infections in healthy children and adolescents aged 2-16 years in Asia and Latin America. J Infect Dis pii: jiw 297.

14. World Health Organization (2016) Dengue vaccine: WHO position paper-July 2016. Weekly epidemiological record 91: 349-364.

15. (2016) Asia Dengue Summit 2016.

16. Thisyakorn U, Capeding RM, Hadinegoro SR (2014) The first tetravalent dengue vaccine is poised to combat dengue. WHO Dengue Bulletin 38: 108112.

17. (2016) ASEAN Dengue Day. 